\title{
Editorial
}

Onkologe 2020 $26: 674-675$

https://doi.org/10.1007/s00761-020-00818-2

(c) Springer Medizin Verlag GmbH, ein Teil von Springer Nature 2020

\author{
Klaus Höffken ${ }^{1} \cdot$ Heinz Schmidberger ${ }^{2}$. Verena Stratmann ${ }^{3}$ \\ 'Berlin, Deutschland \\ ${ }^{2}$ Universitätsmedizin Mainz, Mainz, Deutschland \\ ${ }^{3}$ Springer Medizin Verlag GmbH, Heidelberg, Deutschland
}

\section{Zur Verabschiedung des Herausgebers Prof. Dr. Peter Schlag}

Die Herausgeber und der Springer Medizin Verlag möchten sich von Herrn Prof. Dr. Peter M. Schlag als einem der drei Gründungsherausgeber der Fachzeitschrift Der Onkologe verabschieden. Prof. Schlag verlässt nach über 25-jähriger Mitarbeit das Herausgeberboard.

Die Zeitschrift verabschiedet damit einen der herausragenden deutschen Vertreter der chirurgischen Onkologie als Mitherausgeber und gibt ihm als Editor Emeritus den gebührenden Platz als Gründungsherausgeber.

Prof. Schlag hat die Zeitschrift Der Onkologe durch seine hohe fachliche Kompetenz wesentlich mitgeprägt und dabei über sein eigenes Fachgebiet hinaus stets auf eine abgewogene, interdisziplinäre Betrachtung in den Schwerpunktheften geachtet und großen Wert gelegt. Die Abhandlung wichtiger onkologischer Neuentwicklungen in Schwerpunktheften wurde von ihm mit angeregt und die Aufnahme von neuen Rubriken, wie z. B. die der Psychoonkologie, initiiert.

Mit großem Engagement hat er über die vielen Jahre entscheidend zum Erfolg und Ansehen dieser Fachzeitschrift beigetragen. Wir sind ihm zu großem Dank verpflichtet und werden seine wertvolle Arbeit vermissen!

Mitherausgeber und Verlag bedauern daher den von Prof. Schlag getroffenen Entschluss, aus dem Board der Herausgeber, welchem er derzeit gemeinsam mit Herrn Prof. Höffken und Herrn Prof. Schmidberger vorstand, auszuscheiden. Wir respektieren aber nach so langer und erfolgreicher Tätigkeit für diese Zeitschrift seinen Entschluss und werden uns als Mitherausgeber und Verlag weiter für Qualität und Aktualität dieser Fachzeitschrift einsetzen und damit gemeinsam weiter unser Bestes geben, um die Erwartungen der Leserschaft von Der Onkologe zu erfüllen.

Für die Herausgeber

Prof. Dr. Klaus Höffken

Prof. Dr. Heinz Schmidberger

Für den Springer Medizin Verlag Verena Stratmann

\section{Korrespondenzadresse}

Verena Stratmann

Springer Medizin Verlag GmbH

Tiergartenstr. 17, 69121 Heidelberg,

Deutschland

verena.stratmann@springer.com

Interessenkonflikt. K. Höffken, H. Schmidberger und V. Stratmann geben an, dass kein Interessenkonflikt besteht. 
Hier steht eine Anzeige.

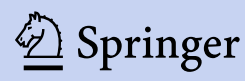

